\title{
Total Shoulder Arthroplasty
}

\author{
Joaquin Sanchez-Sotelo*
}

Department of Orthopedic Surgery, Gonda 14, Mayo Clinic, 200 First Street SW, Rochester MN 55905, USA

\begin{abstract}
Shoulder arthroplasty has been the subject of marked advances over the last few years. Modern implants provide a wide range of options, including resurfacing of the humeral head, anatomic hemiarthroplasty, total shoulder arthroplasty, reverse shoulder arthroplasty and trauma-specific implants for fractures and nonunions. Most humeral components achieve successful long-term fixation without bone cement. Cemented all-polyethylene glenoid components remain the standard for anatomic total shoulder arthroplasty. The results of shoulder arthroplasty vary depending on the underlying diagnosis, the condition of the soft-tissues, and the type of reconstruction. Total shoulder arthroplasty seems to provide the best outcome for patients with osteoarthritis and inflammatory arthropathy. The outcome of hemiarthroplasty for proximal humerus fractures is somewhat unpredictable, though it seems to have improved with the use of fracturespecific designs, more attention to tuberosity repair, and the selective use of reverse arthroplasty, as well as a shift in indications towards internal fixation. Reverse shoulder arthroplasty has become extremely popular for patients with cufftear arthropathy, and its indications have been expanded to the field of revision surgery. Overall, shoulder arthroplasty is a very successful procedure with predictable pain relief and substantial improvements in motion and function.
\end{abstract}

Keywords: Arthroplasty, shoulder, osteoarthritis, shoulder fractures, cuff-tear arthropathy, reverse arthroplasty.

\section{INTRODUCTION}

Over the last few years, shoulder arthroplasty has undergone substantial improvements. Detailed anatomic studies about the morphology of the proximal humerus have facilitated the design of newer modular implant systems that allow a more anatomic humeral head replacement. In patients with osteoarthritis or inflammatory arthropathies and glenoid disease, total shoulder arthroplasty seems to be superior to hemiarthroplasty. The risk of glenoid loosening seems to have decreased with improvements in implant design and surgical technique. For complex proximal humerus fractures, outcomes of hemiarthroplasty have improved due to better patient selection, the development of fracture-specific implants, careful tuberosity reconstruction, and shoulder immobilization for the first few weeks after surgery. Reverse shoulder arthroplasty has emerged as a very attractive alternative for patients with cuff-tear arthropathy, and its indications continue to expand, especially for revision surgery. Complications after shoulder arthroplasty may include infection, instability, neurovascular injury, stiffness, cuff tear, periprosthetic fractures, glenoid erosion and component loosening. The results of revision surgery have continued to improve over time.

\section{THE HUMERAL COMPONENT: FIXATION AND} DESIGN

\section{Fixation}

The fixation and design of the humeral component for anatomic shoulder arthroplasty has improved substantially over time. The component initially designed by Neer was a

*Address correspondence to this author at the Department of Orthopedic Surgery, Gonda 14, Mayo Clinic, 200 First Street SW, Rochester MN 55905, USA; Tel: 507-538-1953; Fax: 507-266-2533;

E-mail: sanchezsotelo.joaquin@mayo.edu monoblock component with a smooth surface. It was initially fixed with polymethylmethacrilate; however, surgeons started to implant this component without cement in patients with good bone quality $[1,2]$. Later studies demonstrated that for this particular component cementless fixation was associated with a high incidence (over $50 \%$ ) of progressive radiolucent lines and/or migration [3, 4], not surprising taking into account that successful cementless fixation usually requires some texturing of the component surface.

Cemented fixation of the humeral component provides several advantages: it is associated with a very low rate of mechanical failure, antibiotics may be added to the cement to prevent infection, and it allows adequate positioning of the humeral component in patients with poor bone quality, proximal humerus fractures, or deformity. However, cementless fixation is very attractive, especially due to the difficulties associated with extracting well-fixed cement from the humeral canal in revision surgery. There are also isolated case reports of iatrogenic radial nerve injuries secondary to cement extrusion through the nutrient artery foramen. Cement pressurization is generally not recommended, and a bone plug should be used to limit distal migration of the cement.

Most modern humeral components are manufactured with surface texturing that has been associated with improved fixation compared to Neer smooth component [5]. There are only a few studies regarding the outcome of cementless fixation in shoulder arthroplasty. Sperling et al. reported a $6 \%$ radiographic mechanical failure rate with the first generation of the Cofield component, which was porous coated only underneath the humeral head. Most current components are manufactured with ingrowth surfaces that extend a variable amount below the humeral head level and provide adequate mid to ling-term fixation [6]. For primary surgery, it is probably best to select a component that does 
not achieve fixation through the whole length of the stem in order to facilitate later revision surgery.

We currently favor cementless fixation of the humeral component for most patients (Fig. 1), and limit cement fixation when it is absolutely required, such as in proximal humerus fractures or when adequate primary stability of the humeral component cannot be achieved even with proximal humerus cancellous bone grafting [7].

\section{Design}

There have been three major generations of anatomic humeral components based on their design. First generation components were monoblocks manufactured in a very limited number of sizes. Second generation components were characterized by the introduction of modular heads and ingrowth coating. Modular heads facilitate selection of the ideal head size for each particular patient in order to balance the soft tissues. They also facilitate revision surgery by allowing removal from the stem. Interestingly, it has been difficult to demonstrate improved clinical outcomes by using modular components [8].

The design of third generation components was prompted by several anatomic studies analyzing the relative variability of some anatomic parameters $[9,10]$. These third generation humeral components are commonly referred to as anatomic or adaptable [11]. Depending on the design used, these components allow adjustments of the prosthetic humeral head position referenced to the stem in the anteroposterior and mediolateral directions. Some implants also allow for various degrees of head inclination. It is important to understand that the terminology used for different systems may change. For example, in the Aequalis system the term offset refers to the relationship between the center of the humeral head and the center of the humeral stem; in the Cofield system, such distance is referred to as eccentricity, whereas offset is used to name prosthetic heads with extra thickness for a given diameter, which would lateralize the humerus compared to the glenoid.

Another interesting option for selected patients is the use of so-called resurfacing implants [12, 13]. Some authors have published a high rate of satisfactory outcomes using resurfacing implants fixed with cement or coated with hydroxyapatite. These components do not have an intramedullary stem, and to a given extent they reproduce anatomically the morphology of the proximal humerus as the cover the native humeral head. They are especially attractive in patients with associated proximal humeral deformity that would otherwise require an associated osteotomy or stem bending. They may also be useful in rheumatoid patients requiring elbow arthroplasty. The stem of the humeral component of the elbow prosthesis may interfere with insertion of the shoulder component or leave a very narrow segment between the two stems, which may increase the risk of a periprosthetic fracture. An additional advantage of resurfacing arthroplasty is an easier revision surgery on the humeral side if it became necessary.

Resurfacing arthroplasty has some disadvantages as well: it may be difficult to achieve adequate stability of the prosthesis if the local bone stock is compromised. In addition, the implantation of a glenoid component is more
(A)

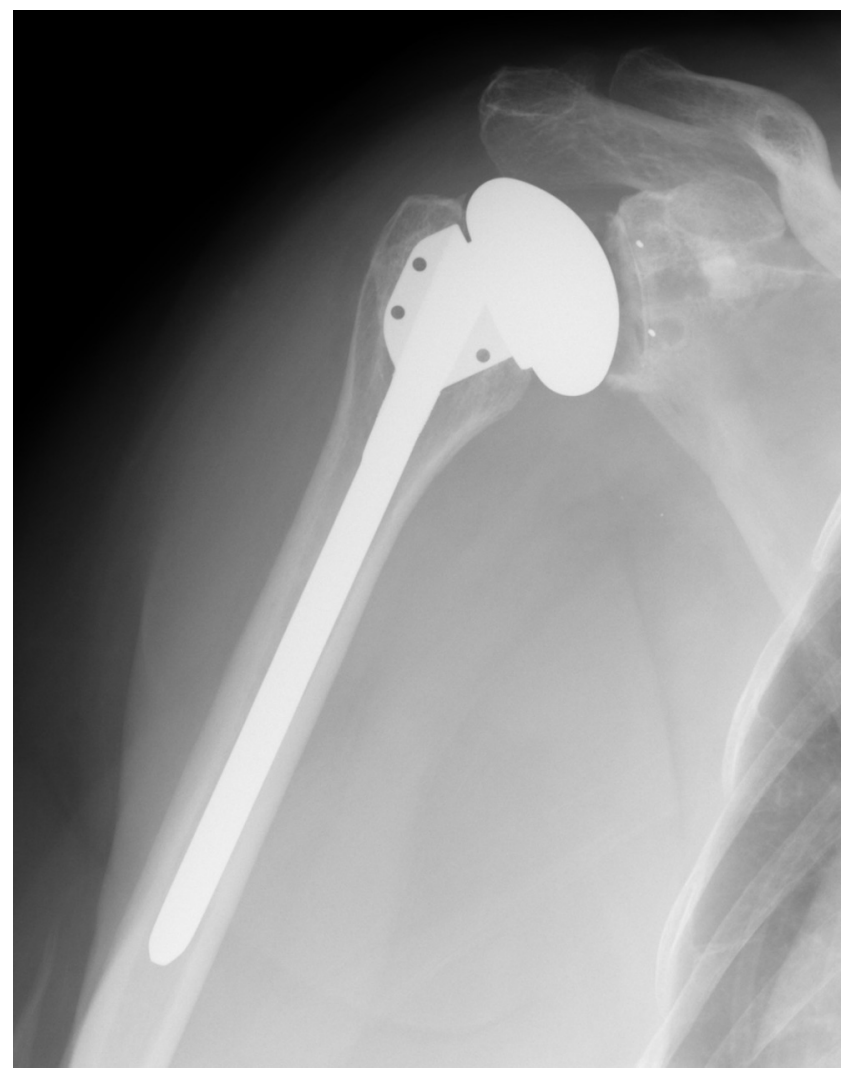

(B)

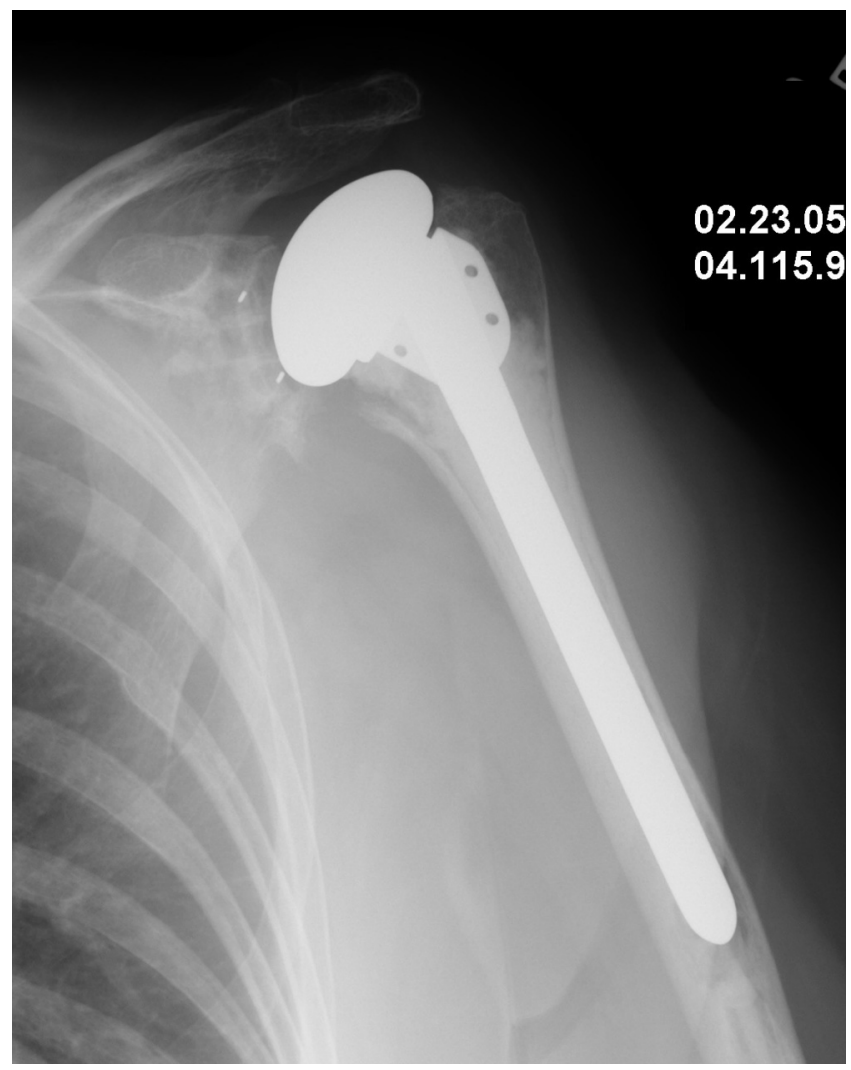

Fig. (1). Anteroposterior radiographs corresponding to two examples of shoulder arthroplasty using cementless (A) and cemented (B) humeral components. 
difficult, as the exposure is limited by preservation of the humeral head. Soft-tissue balance may also be difficult to achieve, as the size and position of humeral head cannot be altered much. Finally, it is difficult to assess the radiographic bone-implant interface.

\section{THE GLENOID COMPONENT: FIXATION, DESIGN AND INDICATIONS}

\section{Fixation and Design}

Most glenoid components are fixed with polymethylmethacrilate. The traditional component is an all-polyethylene implant with a slightly convex backside and a keel to be inserted into the glenoid vault. Most currently used components have their keel replaced by two or more pegs. There are multiple peg configurations. Some components are designed for hybrid fixation, where one of the pegs is designed for ingrowth and fabricated as either a grooved allpolyethylene peg or a metal ingrowth peg. There is interest in so-called self-pressurizing glenoids, with pegs designed to provide and maintain pressure while the cement is curing. Pegged components seem to allow more accurate preparation of the glenoid bone and have been associated in some studies to a lower rate of radiolucent lines in the immediate postoperative radiograph [14]. However, in patients with bone loss it may not be possible to implant a pegged component, and a keeled component may be required (Fig. 2).

Despite improvements in design and cementing techniques, glenoid loosening continues to be a relatively common mode of failure after total shoulder arthroplasty $[15,6]$. Cementless fixation remains an attractive alternative, but unfortunately the first generation of uncemented implants were associated with a high failure rate [16-18]. These components had relatively thin polyethylene inserts in order to avoid overstuffing of the glenohumeral joint, and they failed secondary to catastrophic polyethylene wear or dissociation. New materials such as porous titanium or tantalum may allow manufacturing of more successful cementless glenoid components in the future.

There is some controversy regarding the ideal relationship between the radius of curvature of the prosthetic humeral head and the articular surface of the glenoid component. In general, most surgeons favor a few millimeters of mistmatch, which creates a less constrained joint and may be associated with less radiolucent lines [19].

\section{Indications}

Controversy persists about the indications for implantation of a glenoid component as opposed to a hemiarthroplasty. Some state that total shoulder arthroplasty and hemiarthroplasty provide similar rates of pain relief and satisfactory outcome, and the risk of late glenoid component loosening cannot be neglected. There are other reasons that are not commonly mentioned but probably influence the decision to perform a hemiarthroplasty and not a total shoulder arthroplasty: glenoid exposure is difficult, glenoid implantation requires more assistance and prolongs surgical time, and the difference in reimbursement is not large. However, most current literature clearly shows that for patients with osteoarthritis and rheumatoid arthritis, total shoulder arthroplasty is more reliable in terms of both pain relief and functional improvements [20, 21]. Interestingly, revision of a failed hemiarthroplasty secondary to progressive glenoid erosion seems to be more common than revision of a failed total shoulder arthroplasty for glenoid loosening [22].

(A)

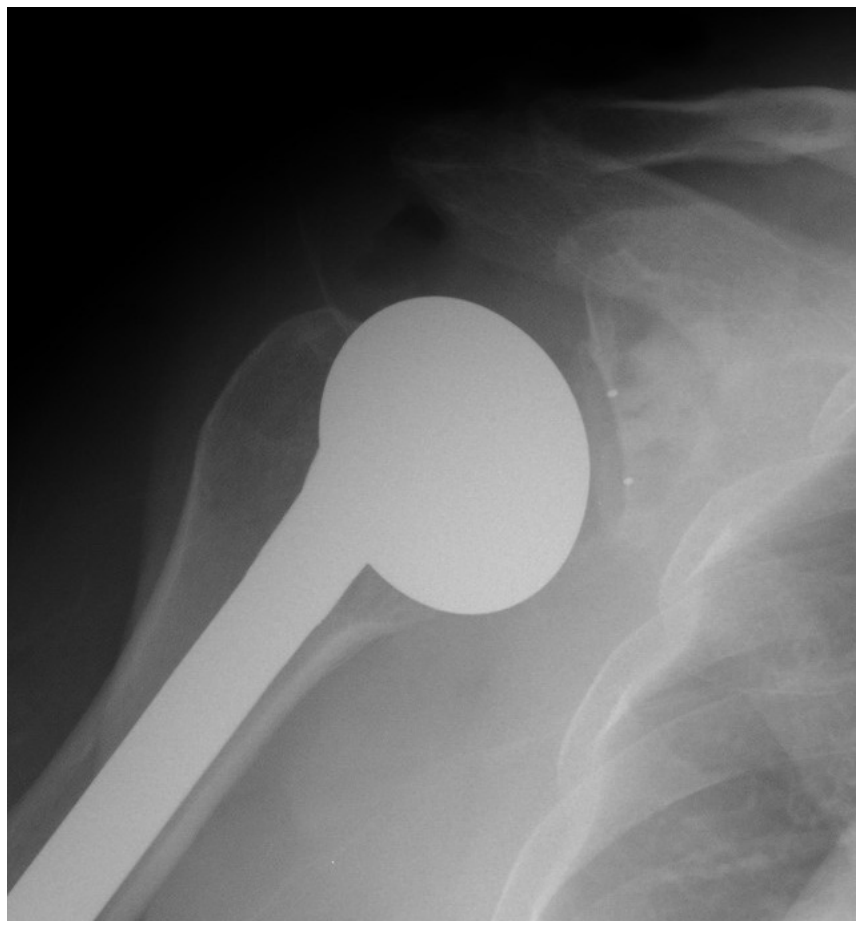

(B)

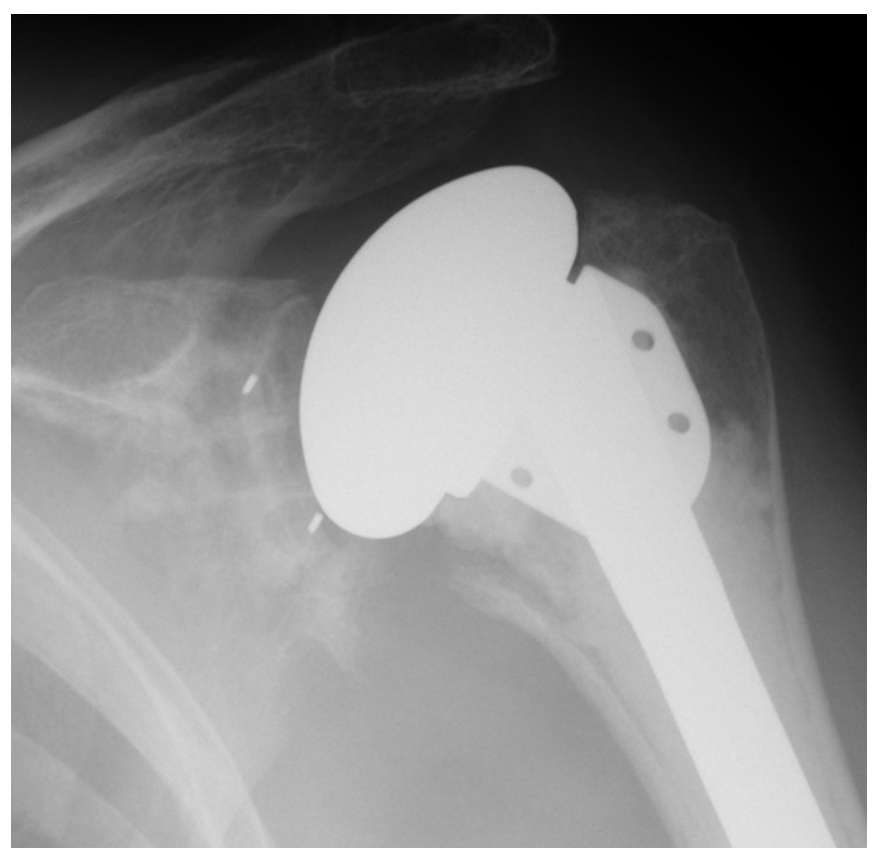

Fig. (2). Radiographs corresponding to cemented pegged (A) and keeled (B) glenoid components. Note the quality of the cementing technique with absence of radiolucent lines.

The main risk factors for glenoid component loosening include poor remaining bone stock, rotator cuff deficiency leading to superior humeral migration (eccentric contact may facilitate glenoid loosening [23]), and a poor cementing 
technique. In patients with a completely normal glenoid articular cartilage (early osteonecrosis, proximal humerus fractures) implantation of a glenoid component is unnecessary. Table 1 summarizes the relative indications of hemiarthroplasty and total shoulder arthroplasty.

Table 1. Indications for Hemiarthroplasty and Total Shoulder Arthroplasty

\begin{tabular}{|l|l|}
\hline Hemiarthroplasty & $\begin{array}{l}\text { Osteonecrosis with a preserved glenoid } \\
\text { Proximal humerus fractures } \\
\text { Cuff-tear arthropathy } \\
\text { Inflammatory arthropathy (rheumatoid) if } \\
\text { Total shoulder arthroplasty }\end{array}$ \\
& $\begin{array}{l}\text { Primary osteoarthritis } \\
\text { Post-traumatic osteoarthritis } \\
\text { Inflammatory arthropathies } \\
\\
\end{array}$ \\
& Osteonecrosis with an affected glenoid \\
\hline
\end{tabular}

\section{RESULTS OF SHOULDER ARTHROPLASTY}

The results of shoulder arthroplasty vary depending on the underlying diagnosis, the condition of the joint and the softtissues at the time of surgery, and the type of reconstruction performed. The results of shoulder arthroplasty in primary osteoarthritis are satisfactory in a large number of patients: pain is improved in over $90 \%$ of the individuals, and their average elevation is usually over 135 degrees. For this particular diagnosis, total shoulder arthroplasty seems to be superior to hemiarthroplasty [24]. This was clearly shown in a prospective randomized study by Garstman et al. [25] and a long-term 15 year study by Sperling et al. [22].

The results of shoulder arthroplasty in osteonecrosis are similar to osteoarthritis. Hattrup and Cofield reported in 88 patients with osteonecrosis who received a hemiarthroplasty or a total shoulder arthroplasty [2]. Pain and satisfaction improved in $80 \%$ of the patients. The results in posttraumatic osteonecrosis and other sequels of proximal humerus fractures are less satisfactory $[26,27]$ Worse results are to be expected when tuberosity osteotomies are required to correct for the underlying deformity.

Shoulder arthroplasty provides satisfactory results in a large number of patients with inflammatory arthritis as well. Sperling et al. reported on 303 consecutive shoulder arthroplasties performed at the Mayo Clinic for inflammatory arthritis [21]. Both hemiarthroplasty and total shoulder arthroplasty provided significant improvements in pain relief and function. Total shoulder arthroplasty was superior to hemiarthroplasty in patients with an intact rotator cuff; it was equivalent to hemiarthroplasty when the cuff was thin or torn. Other authors have reported similar results [28].

The results of hemiarthroplasty in cuff-tear arthropathy are suboptimal. Sanchez-Sotelo et al. reported on 33 consecutive hemiarthroplasties for cuff-tear arthropathy [29]. Pain improved in approximately $70 \%$ of the shoulders, but mean active elevation was only 90 degrees (Fig. 3). As mentioned below, reverse shoulder arthroplasty is now felt by most to be the procedure of choice for patients with cufftear arthropathy.
(A)

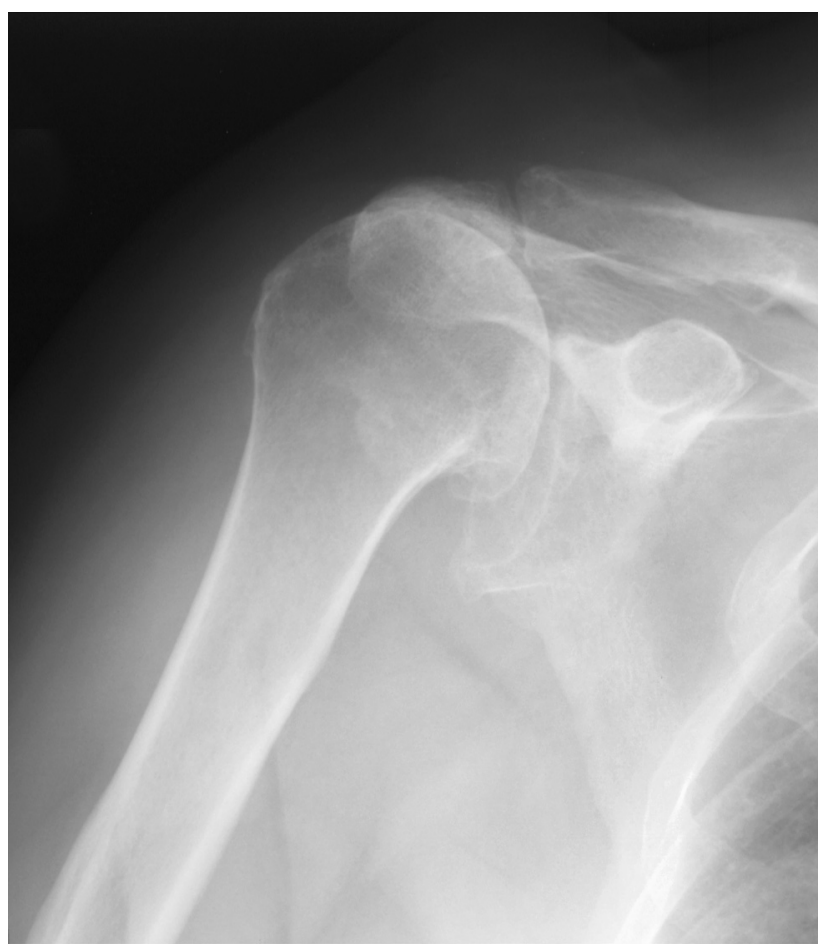

(B)

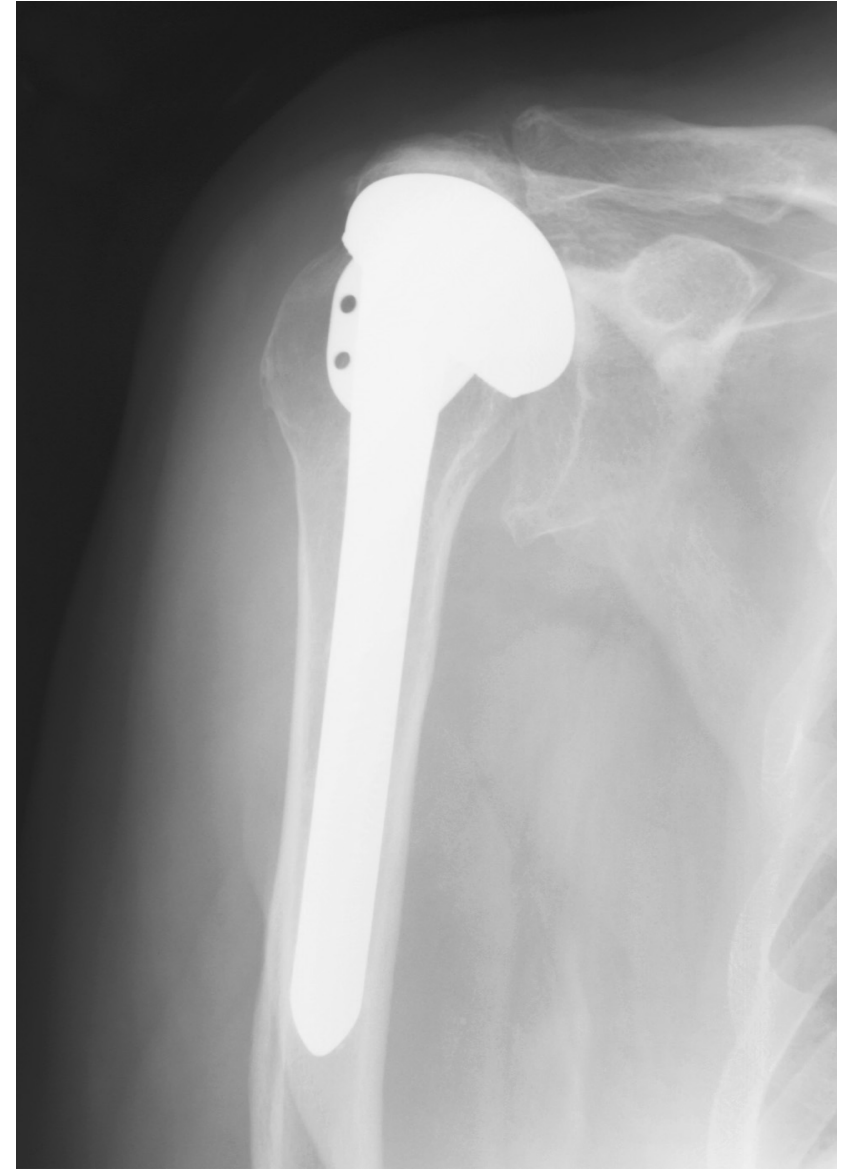

Fig. (3). Radiographs taken before surgery (A) and two years (B) after shoulder hemiarthroplasty for cuff tear arthropathy. 


\section{SHOULDER ARTHROPLASTY IN FRACTURES}

\section{Indications}

The indications for shoulder hemiarthroplasty in fractures has been refined over the last few years due to better understanding of the different fracture patterns, the common use of CT scan for fracture imaging, and improvements in internal fixation devices and techniques. Shoulder hemiarthroplasty is still considered for classic four part proximal humerus fractures, head-impaction fracture and head splitting fractures. Valgus-impacted four part fractures and three-part fractures in the presence of osteopenia were considered in the past indications for shoulder hemiarthroplasty, but are now more commonly treated with internal fixation.

\section{Principles}

Shoulder hemiarthroplasty for proximal humerus fractures is a technically demanding procedure. Most anatomic references used to position the humeral component in shoulder arthroplasty are lost in displaced fractures. In addition, sound tuberosity reconstruction is paramount for the success of this procedure, and it is difficult to achieve. A few general principles are useful in order to obtain a satisfactory outcome:

- The implant selected must facilitate tuberosity reconstruction. Some implants have a very bulky proximal body, which may interfere with tuberosity reduction and healing. Many current systems have dedicated fracture stems with a narrow proximal section and porous or hydroxiapatite coating (Fig. 4).

- $\quad$ Strong tuberosity fixation is required. Most surgeons use a combination of horizontal and vertical heavy non-absorbable sutures through the implant, rotator cuff insertion, and the diaphysis.

- The overall morphology of the proximal humerus needs to be recreated. Most common mistakes include cementing the humeral component too proud or too low and lack of restoration of an adequate headtuberosity relationship.

- Bone autograft from the fractured humeral head should be placed between the tuberosities and the diaphysis, avoiding cement interposition between the fragments.

- $\quad$ The shoulder should be immobilized for a few weeks in some internal rotation; marked internal rotation may place excessive stress on the greater tuberosity.

Tuberosity healing and stability are more important than early motion. Currently, most surgeons favor shoulder immobilization for 4-6 weeks after surgery.

\section{Outcome}

Published results about the outcome of shoulder hemiarthroplasty for proximal humerus fractures have varied tremendously. Neer published a large rate of satisfactory results in his initial study [30]. Most studies published later have reported a higher rate of failure. In the absence of complications, most patients experience little pain, but their motion and strength varies depending on the quality of the reconstruction and tuberosity healing. Average elevation is approximately 90 to 100 degrees, although it may range less than 30 to more than 150 degrees (Fig. 5). The Mayo Clinic experience with shoulder arthroplasty for acute proximal humerus fractures was recently reported in a group of 57 patients followed for a minimum of 5 years [31]. Overall results were considered unsatisfactory in $50 \%$ of the shoulders, and their mean active elevation and active external rotation were 100 and 30 degrees respectively. For these reasons, there is some interest in the use of reverse shoulder arthroplasty for acute proximal humerus fractures.

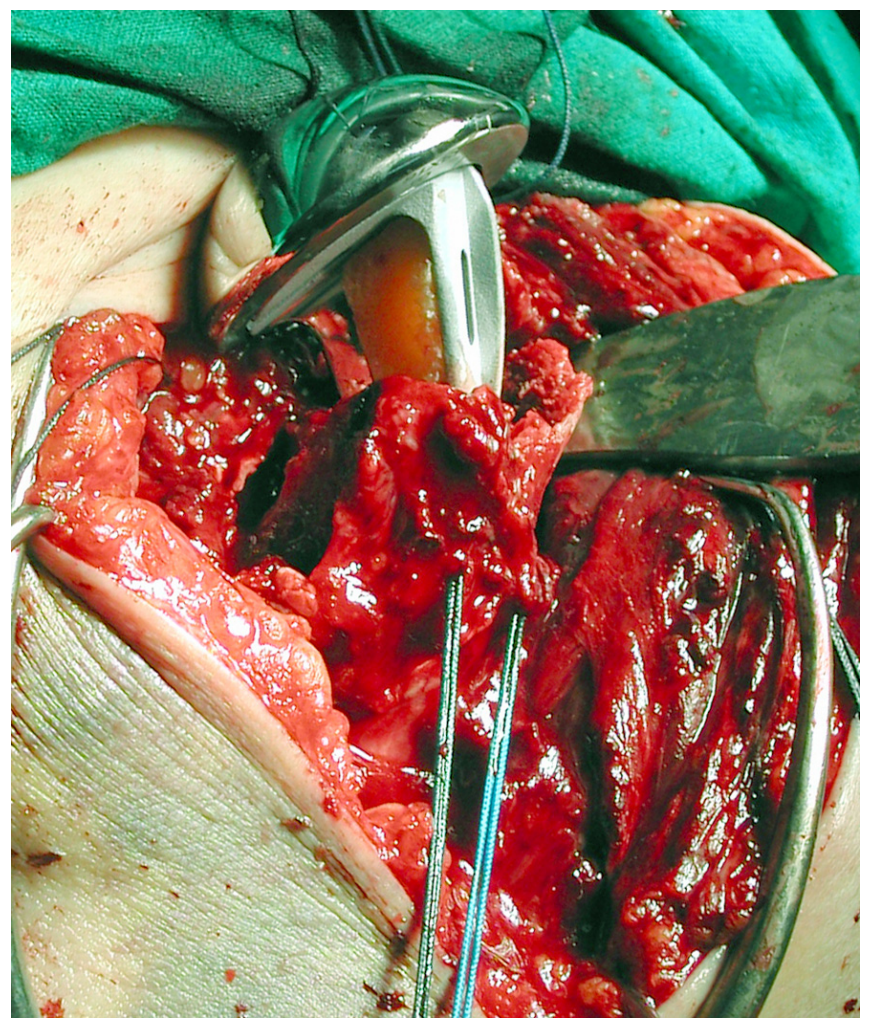

Fig. (4). Dedicated fracture prostheses facilitate proximal humerus reconstruction in complex fractures. This particular implant has a narrow proximal profile, a window for autografting, and hydroxiapatite coating.

\section{REVERSE SHOULDER ARTHROPLASTY}

\section{Rationale}

Reverse shoulder arthroplasty has emerged as a very popular alternative for replacement surgery in the absence of a functional rotator cuff. The term "reverse" refers to the shape of the articulating components: the glenoid component has a convex spherical articular surface, and the articulating portion of the humeral component is a concave polyethylene insert (Fig. 6) [32]. Patients regain active elevation due to the semiconstrained nature of the implant and an improved deltoid lever arm [33]. Other constrained designs used in the past were associated with a high rate of mechanical failure. Reverse arthroplasty has been associated with an acceptable failure rate so far, partly because of better primary stability of the glenoid component and partly due to the medial location of the shoulder center of rotation in reference to the glenoid baseplate. 
(A)

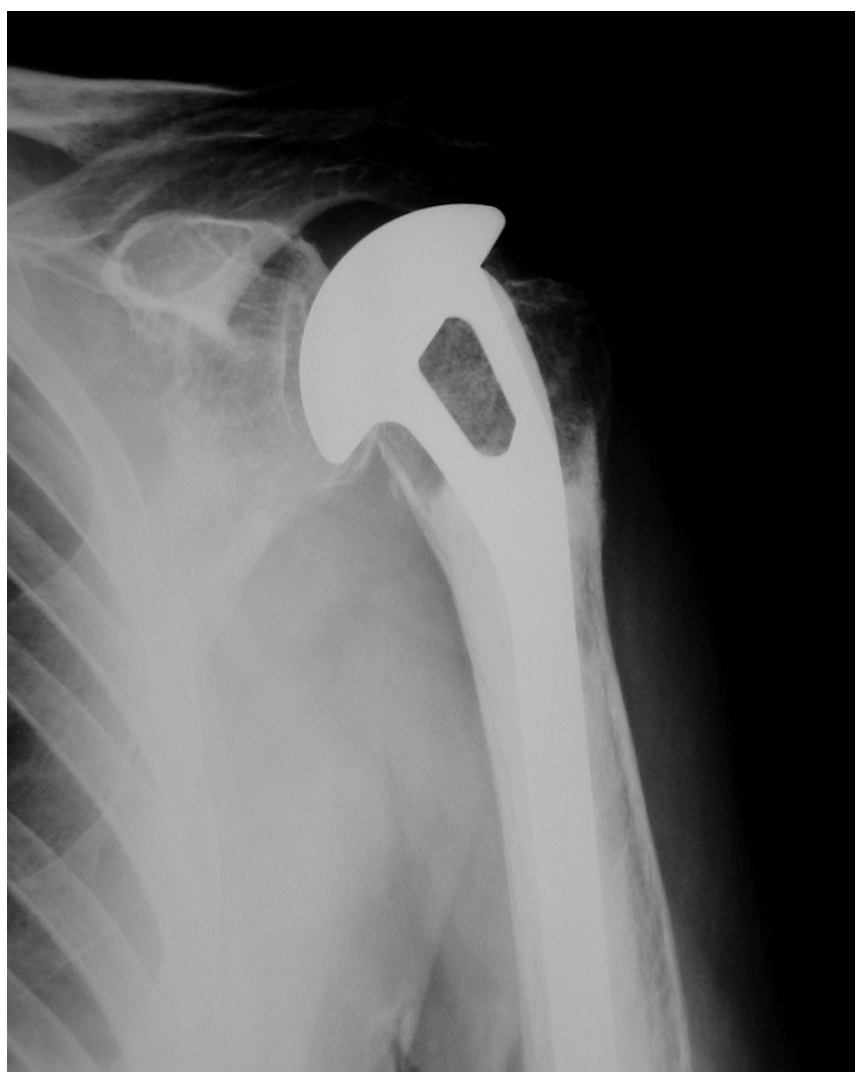

(B)

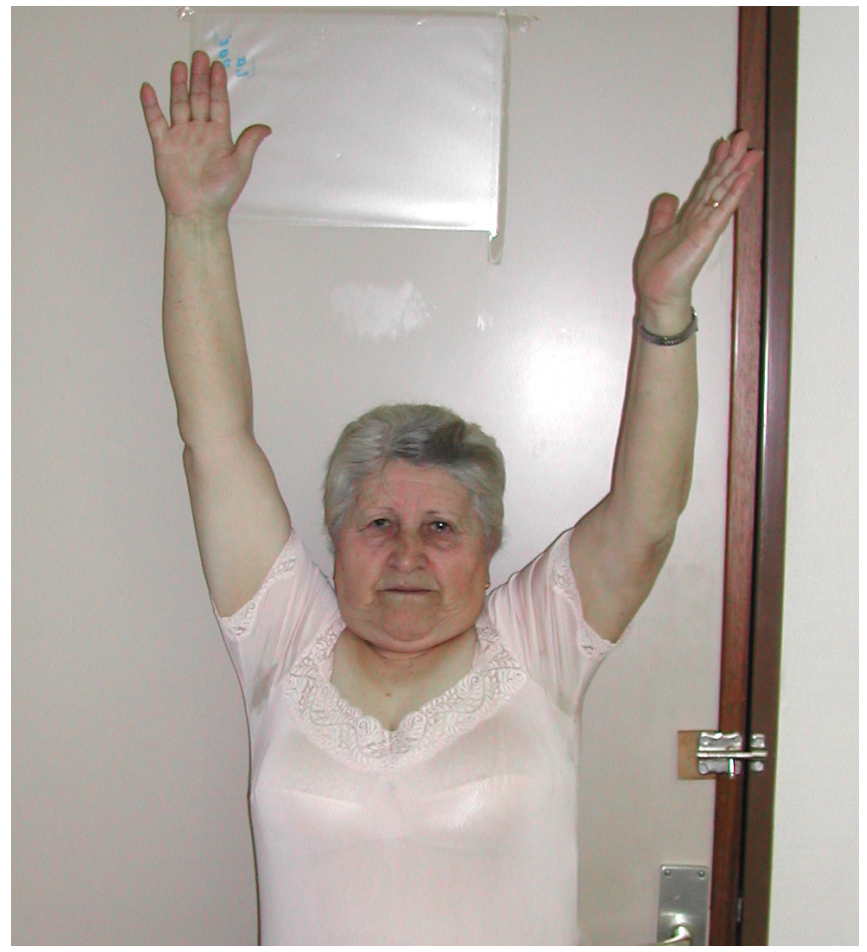

Fig. (5). (A) Postoperative radiograph after hemiarthroplasty and tuberosity reconstruction for proximal humerus fracture. (B), Reasonable function may be achieved provided the reconstruction is adequate and the tuberosities heal.

\section{Indications}

The main indication for reverse shoulder arthroplasty is cuff-tear arthropathy. Reverse shoulder arthroplasty is very successful in this condition, with substantial improvements in pain and active elevation [34]. The indications for reverse shoulder arthroplasty have been expanded to include selected proximal humerus fractures, nonunions and malunions, as well as revision surgery in the presence of gross instability or absence of rotator cuff or tuberosities, reconstruction after resection of tumors, and massive cuff tears with pseudoparalysis but no arthritis [32, 35] (Table 2).

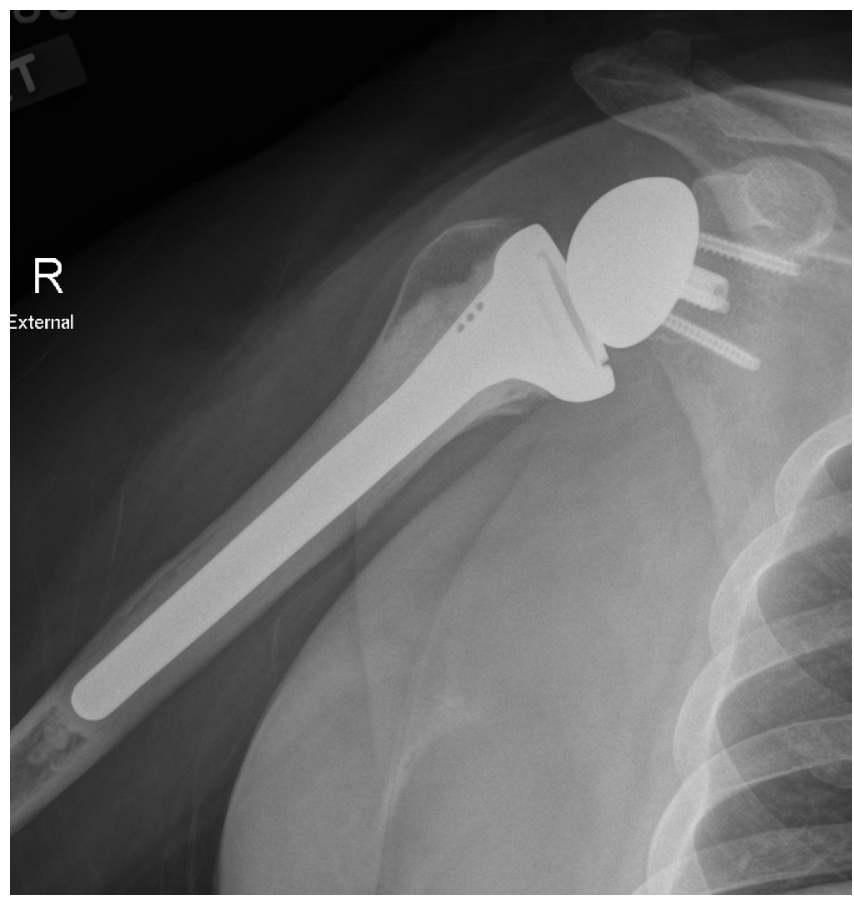

Fig. (6). Anteroposterior radiograph after implantation of a reverse prosthesis.

Table 2. Indications for Reverse Shoulder Arthroplasty

\begin{tabular}{|ll|}
\hline - & Cuff-tear arthropathy \\
\hline - & Massive rotator cuff tear with pseudoparalysis \\
\hline - & Failed shoulder arthroplasty \\
& $\circ \quad$ Absent tuberosities (failed hemiarthroplasty for fracture/nonunion) \\
& $\circ \quad$ Absent cuff (failed hemiarthroplasty for cuff-tear arthropathy) \\
& $\circ \quad$ Instability \\
\hline - & Proximal humerus fractures \\
\hline - & Proximal humerus nonunions \\
\hline - & Reimplantation for deep periprosthetic infection \\
\hline - & Reconstruction after tumor resection \\
\hline
\end{tabular}

\section{Outcomes}

The first large outcome study reporting the results of Dr. Grammont's reverse prosthesis was published by Sirveaux 
et al. in 2004 [36]. These authors reported on 80 patients with a mean follow-up of 3.6 years. The procedure was associated with good pain relief in $96 \%$ of the patients, mean active elevation increased from 73 to 138 degrees, and Constant scores improved from 22 to 65 points. There were five cases of aseptic loosening and seven of glenoid dissociation, and three implants were revised. The integrity of teres minor was found to be essential for recovery of external rotation and was associated with better Constant scores. Slightly worse results were reported by Werner et al. [37], also using the Delta III implant.

The longest follow-up study to date was reported by Guery et al. [38]. These authors reported on 80 prostheses implanted mostly in patients with cuff-tear arthropathy. The 10 -year survivals free of revision surgery and glenoid loosening were $91 \%$ and $84 \%$; however, there was a functional deterioration over time, with less than $60 \%$ survivorship of an absolute Constant score over 30 points. These authors hypothesized occult aseptic glenoid loosening as a possible explanation for the functional deterioration over time.

Several authors have noted the influence of the underlying diagnosis on the expected outcome. Boileau et al. [39], reported on 45 reverse arthroplasties performed for cuff-tear arthropathy (21), sequelae of fracture (5) and revision shoulder arthroplasty (19). The complication rate was $24 \%$, including dislocation (3), deep infection (3), humeral aseptic loosening (1), periprosthetic fractures (3), late acromial fractures (2), and axillary nerve palsy (1). There were better results and fewer complications in the group of patients with cuff-tear arthropathy. Similar findings were reported by Wall et al. [35] in a larger series of 240 reverse shoulder arthroplasties, worse results were reported when reverse arthroplasty was used for revision surgery.

Dr. Frankle's group has reported on the results obtained using a reverse prosthesis with a lateral center of rotation. In 2005 , these authors reported a minimum two-year follow-up study of sixty shoulders with cuff-tear arthropathy [40]. Reverse arthroplasty was associated with statistically significant improvements in pain and function, with a mean active elevation of approximately 105 degrees. However, there was a $17 \%$ complication rate and a $12 \%$ rate of revision for implant failure. Some features of the implant were modified to improve screw fixation of the glenoid component, and the results of the new version of this implant has been reported recently. Cuff et al. [34], reported on 96 shoulders followed for a minimum of 2 years with similar improvements in pain and function, but no cases of either mechanical failure of the glenoid or notching.

\section{COMPLICATIONS AND REVISION SURGERY AFTER SHOULDER ARTHROPLASTY}

\section{Infection}

Infection rates after shoulder arthroplasty are relatively low. However, a relatively large percentage of periprosthetic deep infections after shoulder arthroplasty are difficult to identify preoperatively, as a large number of patients with intraoperative positive cultures do not show abnormalities in their preoperative studies (white cell blood count, sedimentation rate, $\mathrm{C}$ reactive protein, bone scan, aspiration)
[41]. This may be due to the high prevalence of slow growing microorganisms such as Propionibacterium acnes. Two-stage reimplantation is the treatment of choice for most deep infections after shoulder arthroplasty. The rate of reinfection after reimplantation is low, but the functional results are oftentimes compromised by stiffness and cuff dysfunction $[42,43]$.

\section{Instability}

The rate of instability after shoulder arthroplasty is estimated to be $5 \%$. Most instability cases are secondary to a combination of poor component position and soft-tissue imbalance [44]. Anterior instability is associated usually with subscapularis deficiency. Primary tendon repair is associated with a high rate of failure, and allograft augmentation or pectoralis major transfer may be required. Posterior instability usually requires component revision, lengthening of the anterior soft tissue structures, and tensioning of the posterior capsule and rotator cuff. We reviewed the Mayo Clinic experience with revision surgery for prosthetic instability, and found about a $60 \%$ rate of persistent instability after reoperation [44]. Currently, most surgeons favor revising the unstable shoulder arthroplasty to a reverse arthroplasty, but there is not any published report on this particular topic.

\section{Periprosthetic Fractures}

Humeral periprosthetic fractures may happen intraoperatively or after surgery. Intraoperative fractures are especially common in rheumatoid arthritis, and usually happen when the humerus is rotated for exposure. Prevention is key, and may be accomplished by careful and extensive soft-tissue releases before applying any torsion to the humeral shaft, as well as the selective use of the so-called anteromedial approach [45]. Humeral shaft periprosthetic fractures may be treated non-surgically if the implant is well fixed and the fracture line is located at or distal to the tip of the prosthesis. Otherwise, surgery is required and may involve internal fixation with cerclage, plates and/or bone struts with or without revision of the humeral component [46].

\section{Glenoid Complications}

As noted before, the glenoid side of the shoulder joint seems to be much more problematic than the humeral side. On one hand, patients with glenoid arthritis receiving a hemiarthroplasty may continue to experience pain and require revision surgery for placement of a glenoid component [47]. On the other hand, there is a relatively high rate of concerning radiolucent lines around cemented glenoid components. With modern cementing techniques, the rate of glenoid radiographic loosening is probably around 15\% [48]. Failed glenoid components may be revised to a new component provided there is enough remaining glenoid bone stock. Otherwise, the total shoulder arthroplasty needs to be revised to a hemiarthroplasty with bone grafting of the glenoid. Pain relief seems to be better when another glenoid component can be implanted [49].

\section{Other}

Shoulder arthroplasty may be complicated by brachial plexopathy, stiffness, rotator cuff tearing, heterotopic 
ossification and others. Most brachial plexopathies represent transient neuroapraxias that recover over time [50].

\section{SUMMARY}

Shoulder arthroplasty has improved tremendously over the last two decades. Currently, a large array of implants is available for shoulder reconstruction in different conditions. Anatomic total shoulder arthroplasty is the treatment of choice for most patients with primary and posttraumatic osteoarthritis as well as inflammatory conditions of the shoulder. Hemiarthroplasty may need to be considered for patients with no glenoid involvement or severe rotator cuff deficiency. The outcome of hemiarthroplasty for acute proximal humerus fractures or the sequels of trauma seems to be less predictable. Reverse shoulder arthroplasty has emerged as the treatment of choice for patients with cuff-tear arthropathy, and its indications are being expanded to revision surgery and selected proximal humerus fractures and nonunions. Overall, shoulder arthroplasty provides good pain relief to most patients undergoing this procedure; however, these procedures are not devoid of complications, sometimes requiring revision surgery.

\section{REFERENCES}

[1] Cofield RH. Total shoulder arthroplasty with the Neer prosthesis. J Bone Joint Surg Am 1984; 66(6): 899-906.

[2] Hattrup SJ, Cofield RH. Osteonecrosis of the humeral head: results of replacement. J Shoulder Elbow Surg 2000; 9(3): 177-82.

[3] Sanchez-Sotelo J, O'Driscoll SW, Torchia ME, Cofield RH, Rowland CM. Radiographic assessment of cemented humeral components in shoulder arthroplasty. J Shoulder Elbow Surg 2001; 10(6): 526-31

[4] Sanchez-Sotelo J, Wright TW, O'Driscoll SW, Cofield RH, Rowland CM. Radiographic assessment of uncemented humeral components in total shoulder arthroplasty. J Arthroplasty 2001; 16(2): 180-7.

[5] Neer CS $2^{\text {nd }}$, Watson KC, Stanton FJ. Recent experience in total shoulder replacement. J Bone Joint Surg Am 1982; 64(3): 319-37.

[6] Godeneche A, Boileau P, Favard L, et al. Prosthetic replacement in the treatment of osteoarthritis of the shoulder: early results of 268 cases. J Shoulder Elbow Surg 2002; 11(1): 11-8.

[7] Hacker SA, Boorman RS, Lippitt SB, Matsen FA $3^{\text {rd }}$. Impaction grafting improves the fit of uncemented humeral arthroplasty. J Shoulder Elbow Surg 2003; 12(5): 431-5.

[8] Mileti J, Sperling JW, Cofield RH, Harrington JR, Hoskin TL. Monoblock and modular total shoulder arthroplasty for osteoarthritis. J Bone Joint Surg Br 2005; 87(4): 496-500.

[9] Boileau P, Walch G. The three-dimensional geometry of the proximal humerus. Implications for surgical technique and prosthetic design. J Bone Joint Surg Br 1997; 79(5): 857-65.

[10] Hertel R, Knothe U, Ballmer FT. Geometry of the proximal humerus and implications for prosthetic design. J Shoulder Elbow Surg 2002; 11(4): 331-8.

[11] Walch G, Boileau P. Prosthetic adaptability: a new concept for shoulder arthroplasty. J Shoulder Elbow Surg 1999; 8(5): 443-51.

[12] Levy O, Copeland SA. Cementless surface replacement arthroplasty (Copeland CSRA) for osteoarthritis of the shoulder. J Shoulder Elbow Surg 2004; 13(3): 266-71.

[13] Levy O, Funk L, Sforza G, Copeland SA. Copeland surface replacement arthroplasty of the shoulder in rheumatoid arthritis. J Bone Joint Surg Am 2004; 86-A(3): 512-8.

[14] Lazarus MD, Jensen KL, Southworth C, Matsen FA $3^{\text {rd }}$. The radiographic evaluation of keeled and pegged glenoid component insertion. J Bone Joint Surg Am. 2002; 84-A(7): 1174-82.

[15] Torchia ME, Cofield RH, Settergren CR. Total shoulder arthroplasty with the Neer prosthesis: long-term results. J Shoulder Elbow Surg 1997; 6(6): 495-05.

[16] Boileau P, Avidor C, Krishnan SG, et al. Cemented polyethylene versus uncemented metal-backed glenoid components in total shoulder arthroplasty: a prospective, double-blind, randomized study. J Shoulder Elbow Surg 2002; 11(4): 351-9.
[17] Fox TJ, Cil A, Sperling JW, Sanchez-Sotelo J, Schleck CD, Cofield RH. Survival of the glenoid component in shoulder arthroplasty. J Shoulder Elbow Surg 2009; 18(6): 859-63.

[18] Taunton MJ, McIntosh AL, Sperling JW, Cofield RH. Total shoulder arthroplasty with a metal-backed, bone-ingrowth glenoid component. Medium to long-term results. J Bone Joint Surg Am 2008; 90(10): 2180-8

[19] Walch G, Edwards TB, Boulahia A, et al. The influence of glenohumeral prosthetic mismatch on glenoid radiolucent lines: results of a multicenter study. J Bone Joint Surg Am 2002; 84A(12): 2186-91.

[20] Bryant D, Litchfield R, Sandow M, et al. A comparison of pain, strength, range of motion, and functional outcomes after hemiarthroplasty and total shoulder arthroplasty in patients with osteoarthritis of the shoulder. A systematic review and metaanalysis. J Bone Joint Surg Am 2005; 87(9): 1947-56.

[21] Sperling JW, Cofield RH. Total shoulder arthroplasty versus hemiarthroplasty for rheumatoid arthritis: results of 303 consecutive cases. 2007 Annual Meeting of the American Academy of Orthopaedic Surgeons; 2007; San Diego, CA, USA.

[22] Sperling JW, Cofield RH, Rowland CM. Minimum fifteen-year follow-up of Neer hemiarthroplasty and total shoulder arthroplasty in patients aged fifty years or younger. J Shoulder Elbow Surg 2004; 13(6): 604-13

[23] Franklin JL, Barrett WP, Jackins SE, Matsen FA $3^{\text {rd }}$. Glenoid loosening in total shoulder arthroplasty. Association with rotator cuff deficiency. J Arthroplasty 1988; 3(1): 39-46.

[24] Bishop JY, Flatow EL. Humeral head replacement versus total shoulder arthroplasty: clinical outcomes--a review. J Shoulder Elbow Surg 2005 ; 14(1 Suppl S): 141S-6S

[25] Gartsman GM, Roddey TS, Hammerman SM. Shoulder arthroplasty with or without resurfacing of the glenoid in patients who have osteoarthritis. J Bone Joint Surg Am 2000; 82(1): 26-34.

[26] Antuna SA, Sperling JW, Sanchez-Sotelo J, Cofield RH. Shoulder arthroplasty for proximal humeral malunions: long-term results. J Shoulder Elbow Surg 2002; 11(2): 122-9.

[27] Antuna SA, Sperling JW, Sanchez-Sotelo J, Cofield RH. Shoulder arthroplasty for proximal humeral nonunions. J Shoulder Elbow Surg 2002; 11(2): 114-21.

[28] Collins DN, Harryman DT 2nd, Wirth MA. Shoulder arthroplasty for the treatment of inflammatory arthritis. J Bone Joint Surg Am 2004; 86-A(11): 2489-96.

[29] Sanchez-Sotelo J, Cofield RH, Rowland CM. Shoulder hemiarthroplasty for glenohumeral arthritis associated with severe rotator cuff deficiency. J Bone Joint Surg Am 2001; 83-A(12): 1814-22.

[30] Neer CS, $2^{\text {nd }}$. Displaced proximal humeral fractures. II. Treatment of three-part and four-part displacement. J Bone Joint Surg Am 1970; 52(6): 1090-103.

[31] Antuna SA, Sperling JW, Cofield RH. Shoulder hemiarthroplasty for acute fractures of the proximal humerus: a minimum five-year follow-up. J Shoulder Elbow Surg 2008; 17(2): 202-9.

[32] Sanchez-Sotelo J. Reverse total shoulder arthroplasty. Clin Anat 2008; 22(2): 172-82.

[33] Boileau P, Watkinson DJ, Hatzidakis AM, Balg F. Grammont reverse prosthesis: design, rationale, and biomechanics. J Shoulder Elbow Surg 2005; 14(1 Suppl S): 147S-61S.

[34] Cuff D, Pupello D, Virani N, Levy J, Frankle M. Reverse shoulder arthroplasty for the treatment of rotator cuff deficiency. J Bone Joint Surg Am 2008; 90(6): 1244-51.

[35] Wall B, Nove-Josserand L, O'Connor DP, Edwards TB, Walch G. Reverse total shoulder arthroplasty: a review of results according to etiology. J Bone Joint Surg Am 2007; 89(7): 1476-85.

[36] Sirveaux F, Favard L, Oudet D, et al. Grammont inverted total shoulder arthroplasty in the treatment of glenohumeral osteoarthritis with massive rupture of the cuff. Results of a multicentre study of 80 shoulders. J Bone Joint Surg Br 2004; 86(3): 388-95

[37] Werner CM, Steinmann PA, Gilbart M, Gerber C. Treatment of painful pseudoparesis due to irreparable rotator cuff dysfunction with the Delta III reverse-ball-and-socket total shoulder prosthesis. J Bone Joint Surg Am 2005; 87(7): 1476-86.

[38] Guery J, Favard L, Sirveaux F, et al. Reverse total shoulder arthroplasty. Survivorship analysis of eighty replacements followed for five to ten years. J Bone Joint Surg Am 2006; 88(8): 1742-7. 
[39] Boileau P, Watkinson D, Hatzidakis AM, Hovorka I. Neer Award 2005: The Grammont reverse shoulder prosthesis: results in cuff tear arthritis, fracture sequelae, and revision arthroplasty. J Shoulder Elbow Surg 2006; 15(5): 527-40.

[40] Frankle M, Siegal S, Pupello D, et al. The Reverse Shoulder Prosthesis for glenohumeral arthritis associated with severe rotator cuff deficiency. A minimum two-year follow-up study of sixty patients. J Bone Joint Surg Am 2005; 87(8): 1697-705.

[41] Topolski MS, Chin PY, Sperling JW, Cofield RH. Revision shoulder arthroplasty with positive intraoperative cultures: the value of preoperative studies and intraoperative histology. J Shoulder Elbow Surg 2006; 15(4): 402-6.

[42] Meleti J, Sperling JW, Cofield RH. Reimplantation of a shoulder arthroplasty after a previous infected arthroplasty. J Shoulder Elbow Surg 2004; 13(5): 528-31.

[43] Sperling JW, Kozak TK, Hanssen AD, Cofield RH. Infection after shoulder arthroplasty. Clin Orthop Relat Res 2001; (382): 206-16.

[44] Sanchez-Sotelo J, Sperling JW, Rowland CM, Cofield RH. Instability after shoulder arthroplasty: results of surgical treatment. J Bone Joint Surg Am 2003; 85-A(4): 622-31.
[45] Gill DR, Cofield RH, Rowland C. The anteromedial approach for shoulder arthroplasty: the importance of the anterior deltoid. J Shoulder Elbow Surg 2004; 13(5): 532-7.

[46] Kumar S, Sperling JW, Haidukewych GH, Cofield RH. Periprosthetic humeral fractures after shoulder arthroplasty. J Bone Joint Surg Am 2004; 86-A(4): 680-9.

[47] Sperling JW, Cofield RH. Revision total shoulder arthroplasty for the treatment of glenoid arthrosis. J Bone Joint Surg Am 1998; 80(6): 860-7.

[48] Meleti J, Boardman ND, $3^{\text {rd }}$, Sperling JW, et al. Radiographic analysis of polyethylene glenoid components using modern cementing techniques. J Shoulder Elbow Surg 2004; 13(5): 492-8.

[49] Cheung EV, Sperling JW, Cofield RH. Revision shoulder arthroplasty for glenoid component loosening. J Shoulder Elbow Surg. $2008 ; 17(3): 371-5$.

[50] Boardman ND, $3^{\text {rd }}$, Cofield RH. Neurologic complications of shoulder surgery. Clin Orthop Relat Res 1999; (368): 44-53.

(C) Joaquin Sanchez-Sotelo; Licensee Bentham Open.

This is an open access article licensed under the terms of the Creative Commons Attribution Non-Commercial License (http://creativecommons.org/licenses/by$\mathrm{nc} / 3.0 /$ ) which permits unrestricted, non-commercial use, distribution and reproduction in any medium, provided the work is properly cited. 